Peripheral prion pursuit

Adriano Aguzzi
Commentary

See related article, pages $703-708$

Institute of Neuropathology, University Hospital of Zürich, Schmelzbergstrasse 12, CH-8091 Zürich, Switzerland.

Phone: 41-1-255-2107; Fax: 41-1-255-4402; E-mail: adriano@pathol.unizh.ch.

J. Clin. Invest. 108:661-662 (2001). DOI:10.1172/JCI200113919.

Prion diseases are transmissible, fatal neurodegenerative diseases affecting animals and humans (1). The prototypical human prion disease, sporadic Creutzfeldt-Jakob disease (sCJD), is thought to originate in the CNS, and no significant accumulation of the infectious agent (the prion) is found in extracerebral compartments. This may be one of the reasons why human-to-human transmission of sCJD has been essentially confined to neurosurgery, tissue transplants, or, before the advent of recombinant DNA technology, injection of cadaveric pituitary hormones.

The bovine spongiform encephalopathy (BSE) epizootic, however, has spurred many additional concerns (2). BSE is clearly transmissible to humans, and 109 Europeans have - at the time of writing - already succumbed to the human counterpart to BSE, which is called variant Creutzfeldt-Jakob disease (vCJD) (3). Unlike sCJD, BSE prions need to find their way to the CNS, which is the only organ system in which they can produce damage. The process by which prions migrate from the site of inoculation (e.g., the gastrointestinal [GI]tract) to the CNS is called neu- roinvasion and represents a crucial step in the pathogenesis of most naturally occurring prion diseases.

A detailed understanding of the cells and the molecules responsible for neuroinvasion is of utmost importance for public health, since an arguably large fraction of the European population may have been exposed to BSE prions in the last 15 years. Moreover, presumptive rate-limiting steps, if they exist, are likely to represent gratifying targets for postexposure prophylaxis, i.e., for interventions aimed at preventing the clinical outbreak of the disease in persons who have been exposed to BSE prions.

\section{Stages of neuroinvasion}

Some of the players in prion neuroinvasion have already been identified. The march of prions through the body appears to occur in two steps, the first of which could be named lymphoinvasion, while the latter represents neuroinvasion proper (Figure 1 ). While the very first step, the passage through the GI mucosal barrier, has not yet been studied in much detail, it has become clear that prions exploit various components of the immune system to move around the body. Both lymphoinvasion and neuroinvasion depend on the presence of B lymphocytes (4). Because expression of the cellular prion protein by B cells is not required for neuroinvasion (5), it has been proposed that their main function is to enable maintenance of follicular dendritic cells (FDCs) by providing lymphotoxin- $\beta$ to spleen and lymph nodes. Indeed, suppression of lymphotoxin- $\beta$ signaling with a soluble receptor impairs peripheral prion pathogenesis (6). Docking of prions to FDCs is facilitated by complement factors and complement receptors, suggesting that prions may be opsonized (7).

In contrast to our relatively welldeveloped description of prion replication in lymphoreticular organs, we know far less about the rest of the trip to the CNS. Orally administered prions can apparently reach the brain via the parasympathetic vagal nerve (8). However, more indirect paths also exist and may represent the more significant route from the gut to the brain. Thus, following intraperitoneal delivery of prions, disease can be delayed by sympathectomy or can be accelerated by sympathetic hyperinnervation of lymphoreticular organs (9).

\section{Figure 1}

Some cellular and molecular aspects of prion neuroinvasion. Prion diseases such as scrapie manifest as afflictions of the central nervous system. After being taken up orally, prions must cross the epithelial barrier of the gut, probably via gastrointestinal $M$ cells (14). Thereafter, en route to the brain, they colonize several organ systems, taking advantage of multiple cell types to effect neuroinvasion. The experiments reported by Aucouturier and colleagues (12) indicate that dendritic cells (DCs) (but also lymphoid cells) isolated from spleens of prion-infected mice can induce disease when injected intravenously into immunodeficient Rag-1-1- mice, suggesting a possible DC-dependent route to neuroinvasion. Other possible roles of dendritic cells in this disease pathway are discussed in the text.

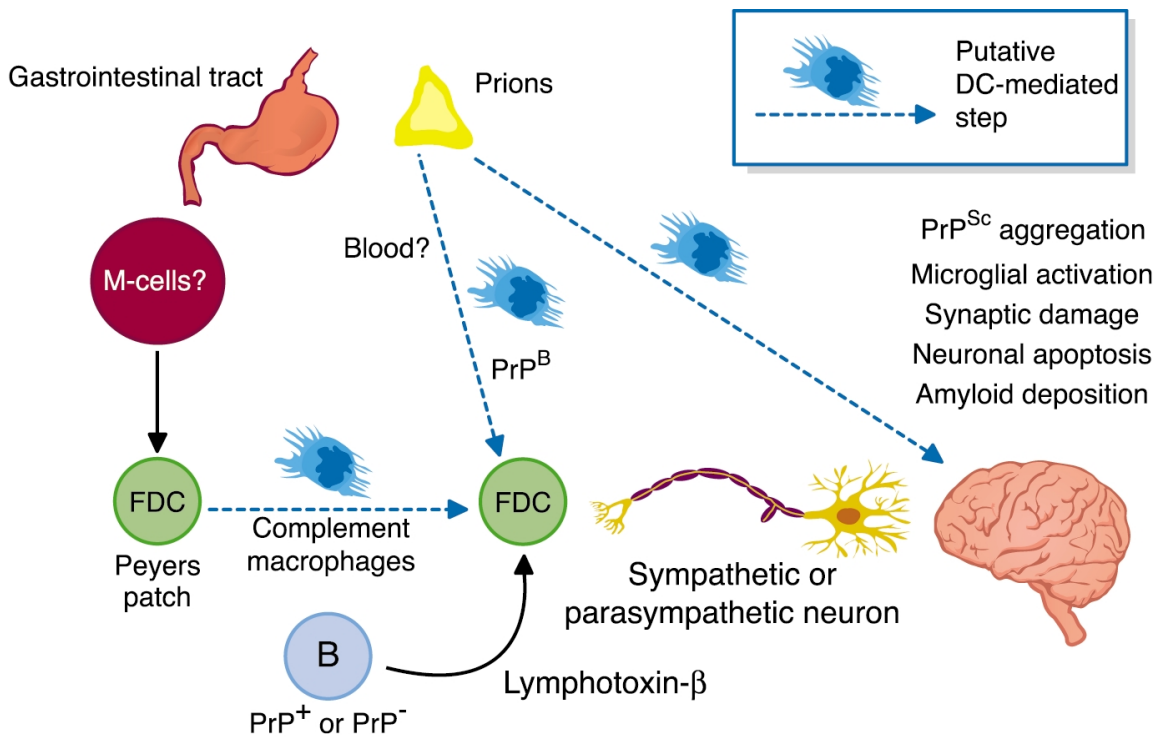


Could prions access the brain by still other means? Hematogenic spread is a possibility worth thinking about, since BSE appears to be transmissible sheepto-sheep by transfusion (10), and several plasma proteins bind prions (11). In the present issue of JCI, Aucouturier and colleagues (12) have investigated whether CD11c $c^{+}$dendritic cells (DCs) may participate in neuroinvasion. DCs are distinct from FDCs: the latter are stromal components of lymphoreticular organs, which are not of hematopoietic origin and, despite the similarity in their names, have nothing in common with DCs - a point of some confusion, even in the published literature on this topic (13). While DCs are professional antigen-presenting cells (APCs), FDCs retain antigens on their surface via Fc $\gamma \mathrm{R}$ and complement receptors, but they do not appear to process antigens, as APCs do.

\section{Potential pathogenic roles for DCs in scrapie}

Aucouturier and colleagues (12) show that isolated DCs from scrapie-infected mice induce disease when injected intravenously in B- and T-cell deficient Rag-1 $1^{-/-}$mice. Their carefully controlled, extensive experiments certainly place DCs on the prion pathogenesis map and show that direct injection of infected cells into the bloodstream can induce prion disease, bypassing a requirement for lymphoinvasion. However, some concerns remain to be addressed before concluding that DCs carry prions to the brain in the course of natural prion infections. For one thing, Rag- $1^{-/-}$mice are not deficient in DCs, yet they are largely protected from peripherally administered prions, indicating that the endogenous pool of DCs does not support neuroinvasion. Therefore, DCs (not unlike B cells) may be necessary but not sufficient for neuroinvasion. In addition, the crucial question of whether depletion of DCs protects host animals from scrapie must still be addressed if one is to assess the relative contribution of DCs to peripheral prion pathogenesis. Finally, the authors report that intravenous injection of other prion-infected mononuclear cell types also leads to scrapie in $R a g-1^{-/-}$mice, a finding that casts some doubts on the specificity of DC-triggered pathogenesis.

Despite these concerns, DCs have been and remain attractive candidates for several prion transport phenomena, including transfer of prions from the basal aspect of mucosal cells (M cells in Figure 1) to Peyer's patches (Figure 1). DCs may well be involved in intranodal trafficking of prions, e.g., from FDCs to sympathetic endings. It will be interesting to determine whether expression of the endogenous prion protein, $\mathrm{PrPC}^{\mathrm{C}}$, is required in this process. Given that $\mathrm{PrP}^{\mathrm{C}}$-expressing hematopoietic cells appear to enhance prion pathogenesis (14), it will be interesting to study whether DCs prepared from $\mathrm{Prnp}^{-/-}$animals will be compromised in their ability to traffic prions to the $\mathrm{CNS}$, relative to $\mathrm{Prnp}^{+/+}$DCs. If the situation modeled by Aucouturier and colleagues (12), lymphoreticular organ-independent spread of prions from the site of entry to the brain, proves to be significant outside of a laboratory setting, manipulation of DCs may indeed add to the growing list of treatments available to arrest the clinical outbreak of vCJD.

1. Aguzzi, A., Montrasio, F., and Kaeser, P.S. 2001 Prions: health scare and biological challenge. Nat. Rev. Mol. Cell Biol. 2:118-126.

2. Weissmann, C., and Aguzzi, A. 1997. Bovine spongiform encephalopathy and early onset variant Creutzfeldt-Jakob disease. Curr. Opin. Neurobiol. 7:695-700.

3. Will, R.G., et al. 2000. Diagnosis of new variant Creutzfeldt-Jakob disease. Ann. Neurol. 47:575-582.

4. Klein, M.A. 1997. A crucial role for B cells in neuroinvasive scrapie. Nature. 390:687-690.

5. Klein, M.A., et al. 1998. PrP expression in B lymphocytes is not required for prion neuroinvasion. Nat. Med. 4:1429-1433.

6. Montrasio, F., et al. 2000. Impaired prion replication in spleens of mice lacking functional follicular dendritic cells. Science. 288:1257-1259.

7. Klein, M.A., et al. 2001. Complement facilitates early prion pathogenesis. Nat. Med. 7:488-492.

8. Beekes, M., McBride, P.A., and Baldauf, E. 1998. Cerebral targeting indicates vagal spread of infec tion in hamsters fed with scrapie. J. Gen. Virol. 3:601-607.

9. Glatzel, M., Heppner, F.L., Albers, K.M., and Aguzzi, A. 2001. Sympathetic innervation of lymphoreticular organs is rate-limiting for prion neuroinvasion. Neuron. 31:25-34.

10. Houston, F., Foster, J.D., Chong, A., Hunter, N., and Bostock, C.J. 2000. Transmission of BSE by blood transfusion in sheep. Lancet. 356:999-1000.

11. Fischer, M.B., Roeckl, C., Parizek, P., Schwarz, H.P., and Aguzzi, A. 2000. Binding of diseaseassociated prion protein to plasminogen. Nature. 408:479-483.

12. Aucouturier, P., et al. 2001. Infected splenic dendritic cells are sufficient for prion transmission to the CNS in mouse scrapie. J. Clin. Invest. 108:703-708.

13. Sy, M.S., and Gambetti, P. 1999. Prion replication-once again blaming the dendritic cell. Nat. Med. 5:1235-1237

14. Kaeser, P.S., Klein, M.A., Schwarz, P., and Aguzzi, A. 2001. Efficient lymphoreticular prion propagation requires $\operatorname{prp}(\mathrm{c})$ in stromal and hematopoietic cells. J. Virol. 75:7097-7106. 